\title{
DESARROLLO DE UN SISTEMA PORTÁTIL DE ANÁLISIS DE CALIDAD DE AGUA
}

\author{
Germán Comina $^{\mathrm{b} *}$, Jackelin Venero ${ }^{\mathrm{b}}$, Hugo Alarcon $^{\mathrm{b}}$ y José L. Solís $\mathrm{s}^{\mathrm{a}, \mathrm{b}}$ \\ ${ }^{a}$ Instituto Peruano de Energía Nuclear, Av. Canadá 1470, Lima, Perú \\ ${ }^{b}$ Facultad de Ciencias, Universidad Nacional de Ingeniería, Casilla 31-139, Lima, Perú.
}

(Recibido Setiembre 05, 2008; Aceptado Noviembre 12, 2008)

\begin{abstract}
A prototype has been developed that could monitor the water quality in real time based on an "electronic tongue". The system is based on electrochemical methods and involves the combination of non-selective sensors (metal electrodes), the signal processing part, and a pattern recognition. It determines attributes (quality, status of a process, etc.) rather than single parameters. Unique response patterns for liquid samples are interpreted with principal components analysis. The prototype is portable and has evaluated the water quality in a rural area (Yaurisque, Cusco). The system could discriminate water with different chemical and bacterological composition.
\end{abstract}

PACS: 07.07.Df; 42.66.-p

Keywords: electronic tongue, E. Coli, principal components analysis.

\section{Resumen}

Se ha desarrollado un prototipo que puede monitorear la calidad del agua en tiempo real que esta basado en una "lengua electrónica". El sistema esta basado en métodos electroquímicos y consiste en la combinación de varios sensores electroquímicos no selectivos (electrodos de metales), el procesamiento y tratamiento de las señales, y un algoritmo de reconocimiento de patrones. Patrones de respuestas únicos para las muestras liquidas son interpretados con un análisis de componentes principales. El sistema determina atributos (calidad, estado del proceso, etc.) antes que parámetros simples. El prototipo es portátil y ha evaluado la calidad del agua de consumo humano en una zona rural (Yaurisque, Cusco). El sistema ha logrado discriminar agua con diferentes composición química y bacteriológica.

Palabras claves: lengua electrónica, E. Coli, análisis de componentes principales.

\section{Introducción}

Contar con agua potable segura es un derecho de toda persona, sin embargo, gran parte de la población mundial no tiene acceso al mismo. En el Perú se tiene agua de baja calidad en las zonas rurales y en la periferia de las zonas urbanas, debido a que no cuentan con redes de agua y desagüe. La contaminación del agua y el limitado acceso al agua potable induce problemas sociales, económicos y de salud. Por ende la incidencia de diarrea infantil y de enfermedades endémicas fatales (hepatitis, fiebre tifoidea o cólera) es muy alta. Si bien es cierto, en zonas urbanas existen adecuados niveles de tratamiento de agua por parte del estado o empresas privadas. En cambio en las zonas rurales los

\footnotetext{
*Correspondig author. e-mail: gcomina@uni.edu.pe
}

pobladores utilizan agua de río, pozo, u otra fuente, que no está caracterizada.

Para determinar la calidad del agua se determina su característica físico química y biológica. En agua libre de contaminación de elementos pesados, los parámetros bacteriológicos del mismo tienen mayor importancia [1]. Los organismos coniformes son buenos indicadores de la calidad higiénica del agua. Las bacterias coliformes, comprenden la estericchia coli y Enterobacter aragenes. El hallazgo de coliformes en el agua indica la polución o contaminación fecal y no es apta para consumo humano. Para analizar la presencia de coliformes totales y coliformes fecales existen diversas pruebas, entre éstas tenemos: el método del número más probable de coliformes (MPN), la filtración a través de una membrana (MF) y la prueba Colilert. El 
método MPN emplea un medio de cultivo líquido en múltiples tubos de ensayos a los cuales se añaden la muestra de agua. Una prueba positiva ocasiona la producción de ácido (color amarillo) y constituye una presunción positiva que requiere confirmación. Mientras que en el método MF, la muestra de agua se pasa a través de un filtro de membrana estéril que retiene las bacterias y luego la membrana se transfiere a la superficie de un medio sólido o a un soporte absorbente, conteniendo el medio de cultivo. Luego se examina la membrana y se cuentan las colonias verdes violáceos con resplandor metálico. En la prueba Colilert se añade una muestra de agua de $100 \mathrm{~mL}$ a un reactivo Colilert. Si hay coliformes, el medio adquiere un color amarillo en $24 \mathrm{~h}$ a $35 \mathrm{C}$. Estos procesos de microbiología demoran por lo menos $18 \mathrm{~h}$. Usando estas técnicas tradicionales no se puede monitorear el agua en tiempo real y un técnico tiene que hacer la medida, lo cual hace que sea inviable de implementarlo en zonas rurales.

Hemos desarrollado un prototipo para el análisis del agua para consumo humano en zonas rurales que esta basado en un sistema conocido como "lengua electrónica" que puede discriminar diferentes muestras líquidas [2-5]. La lengua humana consta de una gran cantidad de células del gusto que están diseminados por la lengua. El líquido o alimento que uno ingiere estimula estas células, estas envían información a través de las neuronas al cerebro donde se compara con patrones almacenados y reconoce el sabor. Imitando los sistemas biológicos, se han desarrollado lenguas electrónicas que son sistemas que determinan atributos del líquido analizado (ver Fig. 1).

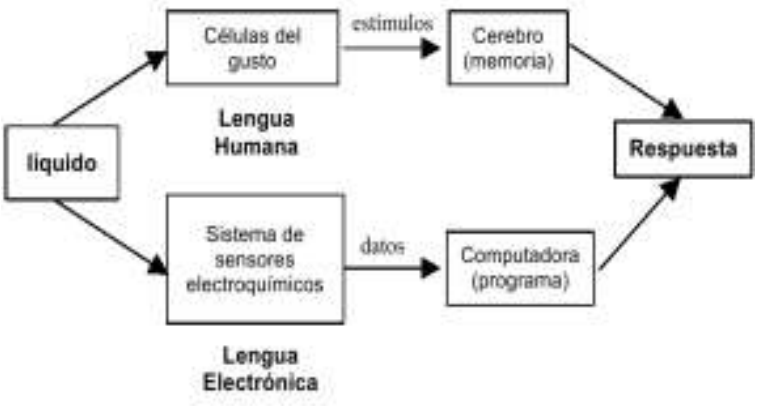

Fig. 1. Esquema de una lengua humana y electrónica.

El sistema desarrollado se basa en métodos electroquímicos [6] y una combinación de sensores no selectivos (electrodos metálicos), un potenciostato que permite hacer crono-amperometría y un algoritmo de análisis de patrones [7].

\section{Prototipo del sistema de análisis de calidad de agua}

El sensor electroquímico utilizado se basa en una celda voltamétrica modificada, donde se tiene electrodos de trabajo de oro y platino, y el contra electrodo y electrodo de referencia es acero inoxidable. Este sistema estará en contacto con el líquido de estudio, y por medio de un potenciostato portátil Uniscan PG580 se le aplica pulsos de potencial y se mide la corriente que pasa a través de cada electrodo de trabajo y el electrodo de referencia. Esta corriente es función de la naturaleza del líquido que se analiza. Utilizando como herramienta el análisis multivariacional (el análisis por componentes principales (PCA)) se puede discriminar líquidos, y también detectar y medir la concentración de un cierto material en el líquido que se analiza $[8,9]$. En la Fig. 2 se observa el diagrama esquemático del prototipo desarrollado.

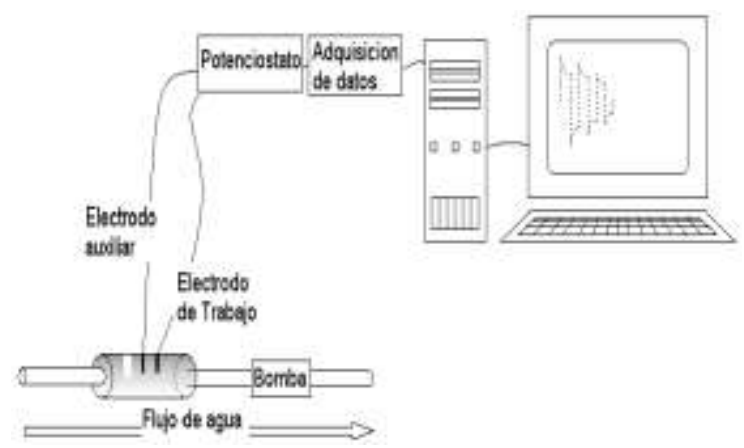

Fig. 2. Diagrama esquemático del sistema de calidad de agua desarrollada.

Los pares de electrodo de trabajo y auxiliar se han diseñado y desarrollado en el laboratorio. Se fabricó un cabezal sensor, ver Fig. 3a, dicho cabezal funciona como soporte para los electrodos de una celda electroquímica de dos electrodos. La base de soporte es de acero inoxidable y funciona como contra-electrodo, los electrodos de trabajo se fabricaron con alambre de oro (Premion $\AA$, $1 \mathrm{~mm}$ de diámetro, con una pureza de $99.985 \%$ ) y platino (World Precision Instruments Inc, $1 \mathrm{~mm}$ de diámetro). El aislante que soporta a los electrodos de trabajo en la estructura del contra-electrodo es acrílico dental (3M ESPE Valux Plus).

Para la toma de muestra de forma automática e incrementar la reproducibilidad en la toma de muestras, se fabricó un mezclador de líquidos con cinco entradas, durante el proyecto solo se usó una entrada. Se ha diseñado una cámara de medida donde se colocará el liquido a analizar, se limpiara y se colocara otro de una forma automática mediante una pequeña bomba. La Fig. 3b muestra la cámara de 
medida y en la tapa se encuentra los electrodos. La cámara de medida se fabrico con Teflon ${ }^{\circledR}$.

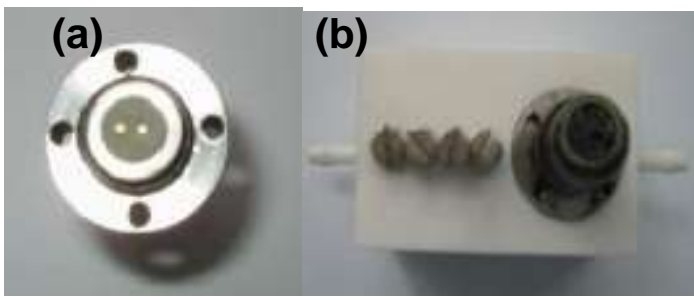

Fig. 3. Fotografías a) de la parte superior del cabezal sensor y b) de la cámara de medida.

La Fig. 4 muestra la fotografía del prototipo desarrollado.

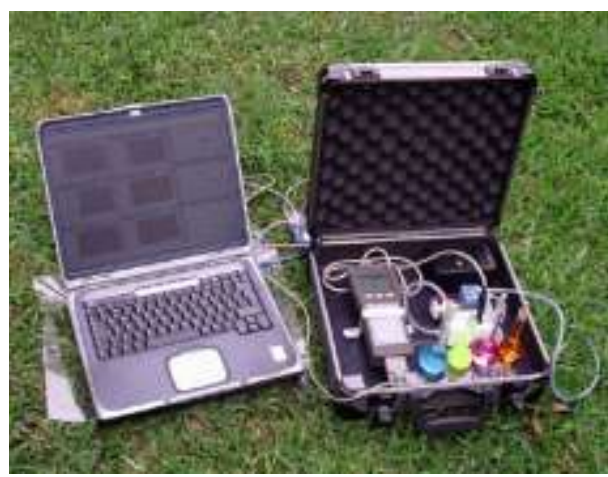

Fig. 4. Foto del sistema desarrollado.

\section{Muestras de agua}

En el presente trabajo se analizaron muestras de agua contaminada con E. coli de tres fuentes diferentes: agua contaminada en el laboratorio, agua contaminada de puquios ubicados en Puente Piedra, Lima, y agua contaminada del río Yaurisque ubicado en Yaurisque, Cuzco.

Los resultados de los análisis microbiológicos fueron realizados en la Laboratorio "Marino Tabusso" de la Universidad Nacional Agraria La Molina de dichas fuentes de agua.

\subsection{Preparación de las muestras de agua contaminada en el laboratorio.}

Se sembró E. coli (ATCC10536) 24 h antes del experimento en placas Petri. La siembra se realizó tomando un inóculo con el Asa de Coll, luego se dispersó el cultivo sobre toda la placa sin dejar espacios vacíos, las placas sembradas se dejaron en la incubadora por un espacio de $18 \mathrm{~h}$. Se colectó $5 \mathrm{~L}$ de agua potable un día antes de cada prueba, para disminuir el nivel de cloro residual en el agua. Al día siguiente se retiró de la incubadora la placa que contiene el cultivo y se dejó a temperatura ambiente por $10 \mathrm{~min}$, luego se tomó una alícuota de $3 \mathrm{~mL}$ del agua colectada y se mezcló suavemente con el cultivo, con la ayuda de una Asa de Coll para preparar un concentrado de bacterias. Se cogió 100 $\mu \mathrm{L}$ de este concentrado y se mezcló con $160 \mathrm{~mL}$ adicionales de agua, esta mezcla se agregó al balde que contiene los $5 \mathrm{~L}$ de agua. En todo momento se trabajó al lado de un mechero Bunsen para crear un ambiente estéril en el área de trabajo.

\subsection{Muestra de agua contaminada de puquíos ubicados en Puente Piedra}

Los puquíos Choqué, Tamboinga y Sauces se encuentran en la zona Industrial, San Remo, y Gallinazos, respectivamente. Todos estos puquíos están en el distrito de Puente piedra.

Los tres puquíos están expuestos sin ningún tipo de protección, los pobladores acceden a estas fuentes de agua de manera directa para abastecerse, incluso entrando con zapatos a dichas fuentes. En algunos casos se observó que bañan a sus mascotas, lavan su ropa, etc. En ninguno de estos puquíos se observa que se protejan los ojos de agua. En esta zona la capa freática es muy próxima a la superficie, y el puquial Choqué se encuentran próximo a un canal de desagüe.

Los resultados del análisis microbiológico (ver Tabla 1) muestran que el agua de estos puquíos que se encuentran contaminadas. La presencia de heterótrofos, y la presencia de coliformes totales es alta, siendo la mas contaminada con estos microorganismos, la muestra del puquial Tamboinga. En estas muestras también se ha encontrado la presencia de coliformes fecales en cantidades considerablemente altas. La presencia de E. coli se encontró en las tres muestras, siendo el agua del puquial Choqué la mas contaminada con E. coli.

Tabla 1. Resultados del análisis microbiológico de muestras de agua de puquial.

\begin{tabular}{llll}
\hline \multicolumn{1}{c}{$\begin{array}{c}\text { Análisis } \\
\text { Microbiológico } \\
\text { (APHA AWWA WEF } \\
-1998)\end{array}$} & $\begin{array}{c}\text { Puquial } \\
\text { Choque }\end{array}$ & $\begin{array}{c}\text { Puquial } \\
\text { Tamboinga }\end{array}$ & $\begin{array}{c}\text { Puquial } \\
\text { Sauces }\end{array}$ \\
\hline $\begin{array}{l}\text { Recuento de } \\
\text { heterótrofos (UFC/ml) }\end{array}$ & $75 \times 10$ & $28 \times 10^{3}$ & $52 \times 10^{2}$ \\
$\begin{array}{l}\text { Enumeración de } \\
\text { coliformes totales } \\
\text { (NMP/100ml) }\end{array}$ & $92 \times 10$ & $24 \times 10^{3}$ & $49 \times 10^{2}$ \\
$\begin{array}{l}\text { Enumeración de } \\
\text { coliformes fecales } \\
\text { (NMP/100ml) }\end{array}$ & $49 \times 10$ & $24 \times 10^{3}$ & $49 \times 10^{2}$ \\
$\begin{array}{l}\text { Enumeración de E-coli } \\
\text { (NMP/100ml) }\end{array}$ & $24 \times 10$ & 21 & $20 \times 10$ \\
\hline
\end{tabular}


Para las pruebas de laboratorio se selecciono el agua proveniente del puquial Choqué debido a que tiene una gran cantidad de E. Coli.

\subsection{Caracterización microbiológica de muestras de agua}

Para la caracterización microbiológica del agua contaminada se utilizo el método de filtración de membrana. Este método se puede utilizar en zonas rurales con un equipo portátil del Agua de Oxfam. Se rotulan 2 frascos que contienen $9.9 \mathrm{~mL}$ de agua destilada estéril para realizar las diluciones. Luego se añade $100 \mathrm{~mL}$ de agua contaminada con la ayuda de una micro pipeta al primer frasco y se mezclan suavemente, esto corresponde a una dilución $10^{-2}$. Posteriormente se vuelve a coger $100 \mathrm{~mL}$ de esta dilución y se agrega al segundo frasco de agua destilada estéril y se mezclan suavemente, esto corresponde a una dilución $10^{-4}$. Esta dilución se filtra con una membrana, luego esta membrana se coloca sobre las placas pad al que se ha añadido 1.75 $\mathrm{mL}$ de Lauril sulfato. Después de colocar la membrana con la muestra en la placa se deja unos minutos al ambiente y luego se lleva a la incubadora a $37^{\circ} \mathrm{C}$ entre 19 y $20 \mathrm{~h}$, al cabo del cual se realiza el conteo o lectura de las unidades formadoras de colonia por $10 \mathrm{~mL}$ (UFC/10mL). Expresando el número de colonias por la dilución a la que se ha trabajado.

La esterilización del equipo de filtración se realizo por la incineración con alcohol metílico, y para desinfectar superficies y otros se usa alcohol etílico. Si el número de UFC/10 mL exceden a 800 se considera incontable como lo indica el método. En todo el trabajo se ha tenido especial cuidado con la limpieza e higiene del material y el ambiente de trabajo.

\subsection{Muestra de agua de una zona rural, Yaurisque, Cusco}

El prototipo fue probado en una zona rural. Este trabajo experimental de campo se realizo en el distrito de Yaurisque a unos $48 \mathrm{~km}$ de la ciudad de Cusco. El lugar esta ubicado a una Altitud de 3330 msnm en la cual el agua de consumo humano no tiene ningún tipo de tratamiento.

Sobre el río Yaurisque desembocan los desagües de la posta médica y del colegio del lugar, el trabajo se ha llevado acabo en colaboración con el Centro de Capacitación de Desarrollo, CECADE, de Cusco.

\section{Resultados y Discusión}

Inicialmente se realizaron las pruebas de voltametría cíclica a muestras de agua limpia y contaminada artificialmente para determinar el rango de voltaje en el cual tienen diferente respuesta. El agua contaminada artificialmente tiene un número de UFC/10 mL que exceden a 800, por ende se considera incontable como lo indica el método, se uso un agua muy contaminada para ver si se puede diferenciarla respecto del agua limpia.

Se eligió un rango de trabajo entre $0.8 \mathrm{~V}$ y $-0.8 \mathrm{~V}$ para evitar la descomposición del agua y una velocidad de barrido de $20 \mathrm{mV} / \mathrm{s}$, los voltagramas obtenidos para el agua limpia y contaminada (ver Fig. 5) nos muestran una marcada diferencia (un corrimiento en valores de corriente) entre $-0.1 \mathrm{~V}$ y $0.4 \mathrm{~V}$. Las medidas de las muestras de agua contaminada se midieron después de 2 h 30 min y $3 \mathrm{~h}$ 30 min de preparada la muestra. Se puede observar que las medidas son reproducibles.
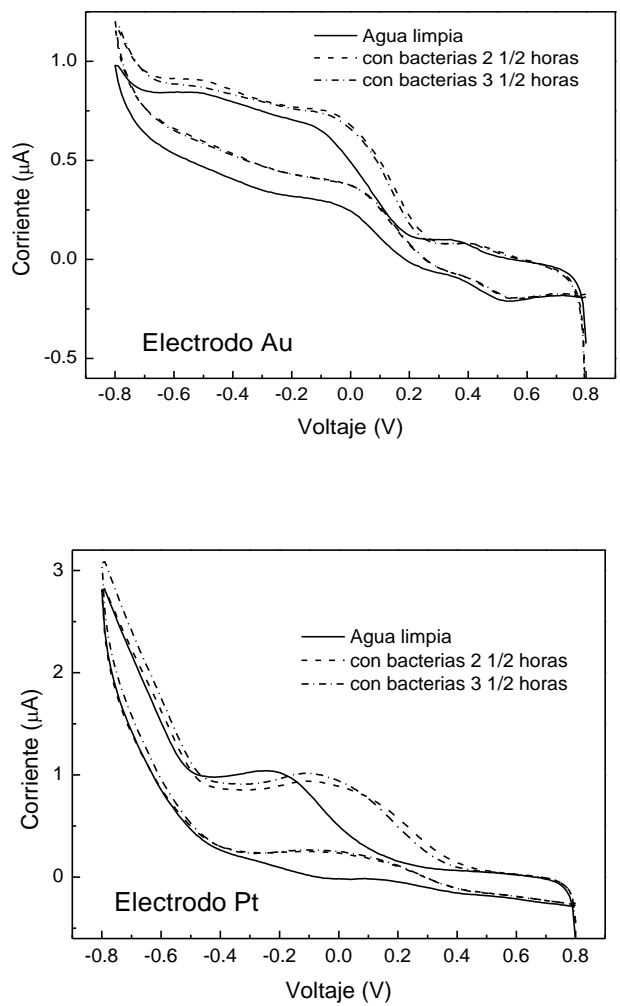

Fig. 5. Voltametria cíclica del agua limpia y contaminada con $E$. coli para el electrodo de oro (a) y patino (b).

A partir de la voltametria cíclica se eligió un potencial escalón como se muestra en la Fig. 6, para el análisis de agua.

Usando este voltaje escalon se puede observar la diferencia entre el agua limpia y el agua contaminada con $E$. coli, los voltajes establecidos fueron de $-0.3 \mathrm{~V}$ $\mathrm{y}$ de $-0.4 \mathrm{~V}$ por un tiempo de $2 \mathrm{~s}$. Se prepararon tres muestras con diferentes concentraciones de E. coli, como se muestra en la Tabla 2. 
Se midió tres veces cada una de las tres muestras en orden aleatorio usando el potencial escalón que se observa en la Fig. 6. En la Fig. 7a se observa las corrientes de respuesta de los electrodos de Au y Pt a las diferentes muestras de agua contaminada artificialmente, solamente con el electrodo de $\mathrm{Au}$ se puede diferenciar claramente las muestras. Para una mejor visualización se obtuvo la gráfica de componentes principales para del electrodo de $\mathrm{Au}$ (ver Fig. 7b).

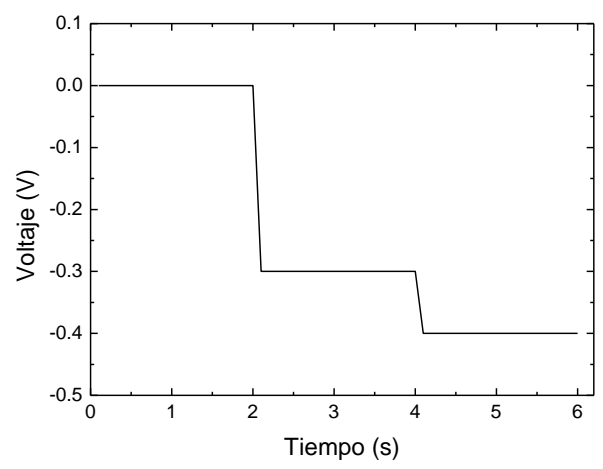

Fig. 6. El voltaje en función del tiempo utilizado para el análisis de agua.

Tabla 2. Concentraciones de las muestras de agua contaminadas en laboratorio.

\begin{tabular}{lccc}
\hline Muestra & M3 & M2 & M1 \\
\hline $\begin{array}{l}\text { Concentración } \\
\text { de E.coli }\end{array}$ & Alta & Media & Baja \\
UFC/ 10 ml & $\begin{array}{l}\text { Incontables } \\
\times 10^{-1}\end{array}$ & $\begin{array}{l}\text { Incontables x } \\
10^{-2}\end{array}$ & $554 \times 10^{-4}$ \\
\hline
\end{tabular}

Una forma económica de desinfectar agua es por medio del uso de la radiación solar, esta técnica se conoce como descontaminación de agua en unidades independientes (DSAUI) [10, 11]. Esta técnica consiste en la irradiación solar de agua contaminada contenida en botellas de plástico (recipientes de bebidas comerciales), obteniéndose al final del proceso agua apta para consumo humano.

Para evaluar nuestro sistema desarrollado, empleamos agua del puquial de Choque, de Puente Piedra; que esta contaminada con E. coli, y con bacterias del género Pseudomonas sp. Tres muestras de agua colocadas en botellas de polietilenotereflatato (PET) de $500 \mathrm{~mL}$ fueron expuestas a la radiación solar durante $4 \mathrm{~h}$ (10 a.m a 2 p.m). Se monitoreo el proceso de desinfección del agua contaminada cada hora, los resultados obtenidos con el sistema desarrollado se muestra en la Fig. 8. Se observa que las muestras se pueden discriminar fácilmente, el prototipo puede monitorear el proceso de desinfección solar. La muestra inicial tenia $42 \mathrm{x}$ $10^{-1}$ UFC/10 mL de E.coli y al cabo de $4 \mathrm{~h}$ de irradiación solar no tiene E. coli.

Una vez que el sistema ha sido optimizado con pruebas de laboratorio se procedió a realizar medidas en una zona rural, Yaurisque, Cusco. Las muestras de agua del río Yaurisque en botellas PET se expusieron al sol por $4 \mathrm{~h}$. La desinfección del agua se logro monitorear con el prototipo. Las muestras colectadas del río Yaurisque fueron analizadas microbiologicamente y muestran una gran contaminación de E.coli (ver Tabla 3). Estas disminuyen después de ser irradiadas con luz solar y después de $4 \mathrm{~h}$ presentan aun una pequeña cantidad de E. coli.
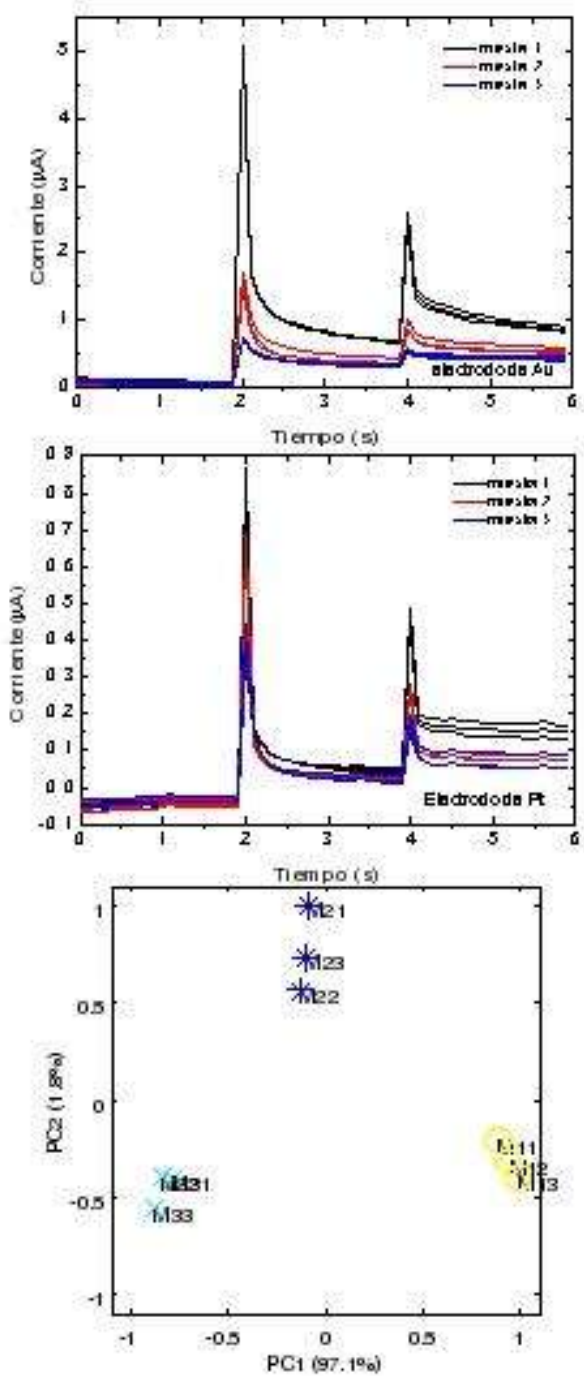

Fig. 7. a) La respuesta de corriente en función del tiempo para las muestras de agua contaminada para los electrodos de $\mathrm{Au}$ y $\mathrm{Pt}$, y b) la gráfica de componentes principales para el electrodo de $\mathrm{Au}$. 
En la Fig. 9 se muestra la respuesta típica de las muestras de agua del río inicialmente y después de ser irradiada con luz solar por $1 \mathrm{~h}, 2 \mathrm{~h}, 3 \mathrm{~h}, \mathrm{y} 4 \mathrm{~h}$. Se puede distinguir las muestras pero no fácilmente. Para lo cual se realizo el análisis de componentes principales, el cual ayuda a observar las diferencias que tienen las muestras de agua de río.
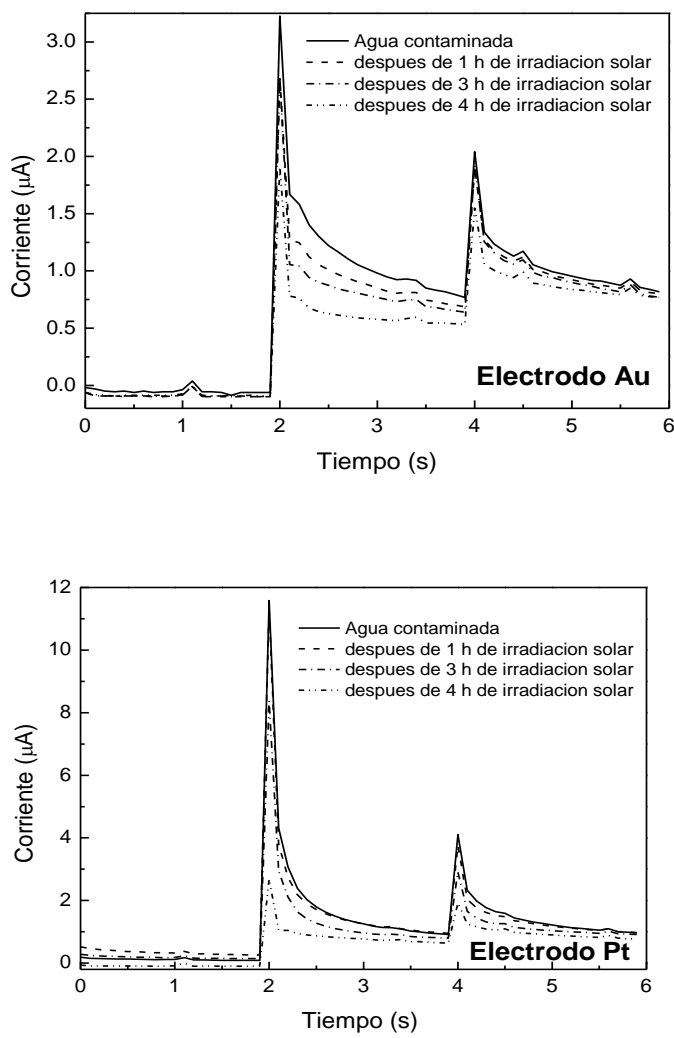

Fig. 8. La respuesta de corriente en función del tiempo para las muestras de agua contaminada para los electrodos de Au y Pt, sometidas diferentes tiempos de irradiación solar.

Tabla 3. Análisis microbiológico de las muestras de agua del río Yaurisque.

\begin{tabular}{ll}
\hline Tiempo & UFC/10 ml \\
\hline Control inicial & $117 \times 10$ E.coli \\
& $190 \times 10$ Pseudomonas sp. \\
$\mathbf{T}_{\mathbf{1}} \mathbf{1 ~ h}$ & $60 \times 10$ E. coli \\
$\mathbf{T}_{\mathbf{2}} \mathbf{2} \mathbf{h}$ & $71 \times 10$ Pseudomonas sp. \\
$\mathbf{T}_{\mathbf{3}} \mathbf{3} \mathbf{h}$ & $19 \times 10$ E.coli \\
$\mathbf{T}_{\mathbf{4}} \mathbf{4 h}$ & $4 \times 10$ E. coli \\
\hline
\end{tabular}

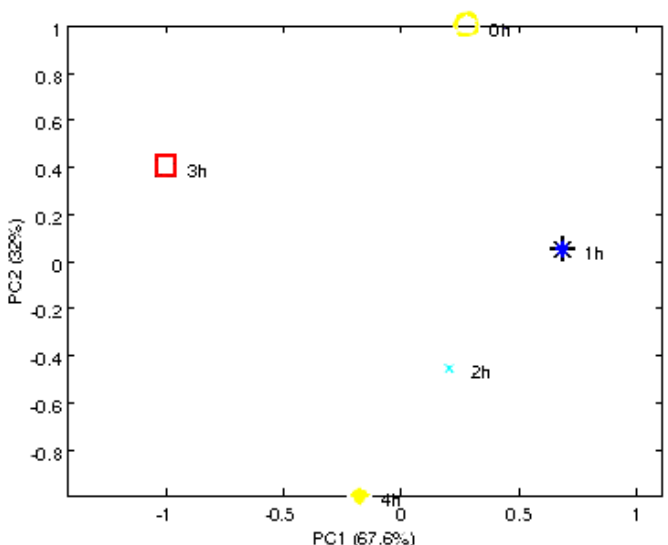

Fig. 9. Gráfica de componentes principales para el electrodo de Au de muestras de agua del río Yaurisque con diferentes tiempos de irradiación solar.

\section{Conclusiones}

El prototipo desarrollado ha logrado discriminar diferentes muestras de agua contaminada con E.coli.

Se ha encontrado que las muestras de agua necesitan analizarse desde el punto de la voltametria cíclica para optimizar el potencial escalón adecuado.

El prototipo desarrollado puede usarse en zonas rurales, puesto que con una pequeña batería se puede alimentar todo el sistema, una medida toma de dos a tres minutos, así que se puede realizar una gran cantidad de medidas.

El potenciostato portátil es la componente que mas caro del equipo, así que esta parte será reemplazado por potenciostato basado en una tarjeta de adquisición usando el USB.

\section{Agradecimientos}

Al CONCYTEC y al Instituto de Investigación de la Facultad de Ciencias de la Universidad Nacional de Ingeniería por la ayuda económica para la realización del trabajo.

\section{References}

[1]. L.M.L. Nollet (Ed.), Handbook of Water Analysis (Food Science and Technology), CRC, Nueva York, (2000).

[2]. F. Winquist, P. Wide, and I. Lundstrom, "An electronic tongue based on voltammetry", Analytica Chimica Acta, 357 (1997) 21-31.

[3]. S. Holmin, Liquid sensing: Development and characterization of an electronic tongue based on electrochemical methods, (2002), Tesis doctoral, Universidad de Linköping University, 
Suecia.

[4]. F. Winquist, C. Krantz-Rülcker, P. Wide, and I. Lundstrom, "Monitoring of freshness of milk by an electronic tongue on basis of voltammetry", Measurement Science and Technology, 9 (1998) 1937-1946.

[5]. G. Comina, S. Holmin, P. Ivarsson, F. Winquist, C. Krantz-Rülcker, "COD monitoring of waste water using an electronic tongue", $2^{\text {nd }}$ SENSPOL Workshop Response To New Pollution Challenges, King's College, London, UK. 4-7 June (2002).

[6]. A.J. Bard, y L.R. Faulkner, "Electrochemical Methods : Fundamentals and Applications", (2000) $2^{\text {nd }}$ ed., Wiley Text Books, EEUU.

[7]. K. Esbensen, "Multivariate Data Analysis in Practice". CAMO Process AS, (2001).

[8]. G.Comina, J.Solis, y W.Estrada, Uso del análisis multivariado de datos y espectrometría de absorción UV-Visible para la determinación simultanea de la concentración de $\mathrm{Co}, \mathrm{Cu}$ y $\mathrm{Ni}$ en una solución, TECNIA 14 (2) (2004) 57-62.

[9]. G.Comina, Supervisión In-Situ y en tiempo real de una muestra líquida usando PCA-3D, REVCIUNI 10 (1) (2006) 18-22.

[10]. D. F. Ollis and H. Al-Ekabi (eds.), Purification and Treatment of Water and Air, Elsevier, Amsterdam, The Netherlands,( 1993).

[11]. P. Galarza, E. Sánchez, D. Maurtua, J. Rodríguez, J. Solis y W. Estrada, Desinfección Solar de Aguas en Tambo Pariachi, Huaycán, Lima, Perú in Desinfección Solar de Aguas en Comunidades Rurales de América Latina, Informe Proyecto OEA AE 141/2001, Organización de Estados Americanos (AICD), Marzo de 2003. M. Litter, H. Mansilla (Eds.), Digital Grafic, La Plata, Argentina, 2003. 